\title{
Medical therapy versus percutaneous coronary intervention in ischemic heart disease: A cost-effectiveness analysis
}

\author{
Aziz Rezapour ${ }^{1}$, Nader Tavakoli ${ }^{2}$, Sadaf Akbari ${ }^{2}$, Marjan Hajahmadi ${ }^{3}$, Hosein Ameri ${ }^{4}$, Reza Mohammadi ${ }^{5}$, \\ Saeed Bagheri Faradonbeh* ${ }^{1}$ (D)
}

Received: 2 Dec 2019

Published: 16 Nov 2020

\begin{abstract}
Background: Ischemic heart disease is categorized into two acute and chronic groups, and its treatments include revascularization and medical therapy. The aim of this study is to evaluate the economic burden of medical therapy compared to percutaneous coronary intervention in ischemic heart disease.

Methods: This study has been done in two steps. The first was a systematic review and meta-analysis to measure the effectiveness of two interventions and the second step was a cost-effectiveness analysis from the perspective of society. The data analysis included a meta-analysis and the Markov cohort simulation. RewMan v5 and tree age software were utilized. Uncertainties related to the model parameters were evaluated using one-way and two-way sensitivity analyses.

Results: Regarding the effectiveness of interventions, the odd ratio of the quality of life in the medical therapy group (CI: 0.76-1.10) was 0.91 times the PCI group ( $\mathrm{p}=0.34)$. This rate for mortality in medical therapy (CI: 0.52-9.68) was 2.23 times more than the PCI group; this result was not significant $(\mathrm{p}=0.02)$. In the cost-effectiveness analysis, the cost-effectiveness threshold was \$ 16,482; ICER in increasing the QoL and reduction in the mortality rate was \$ 25320.11 and \$ 562.6691, respectively. Regarding the sensitivity analysis, the model was not sensitive in changing parameters in a specific domain.

Conclusion: According to this study, PCI is more cost-effective than medical therapy in the reduction of mortality rate and in the field of increasing quality of life. MT strategy is more cost-effective than the PCI. This study considers controversies regarding the most appropriate treatment for patients with ischemic heart disease that is helpful for health policymalkers, cardiologists and health managers.
\end{abstract}

Keywords: Medical therapy, Percutaneous coronary intervention, Ischemic heart disease, Cost-effectiveness analysis

Conflicts of Interest: None declared

Funding: Iran University of Medical Sciences

${ }^{*}$ This work has been published under CC BY-NC-SA 1.0 license.

Copyright $@$ Iran University of Medical Sciences

Cite this article as: Rezapour A, Tavakoli N, Akbari S, Hajahmadi M, Ameri H, Mohammadi R, Bagheri Faradonbeh S. Medical therapy versus percutaneous coronary intervention in ischemic heart disease: A cost-effectiveness analysis. Med J Islam Repub Iran. 2020 (16 Nov);34:155. https://doi.org/10.47176/mjiri.34.155

\section{Introduction}

Ischemic heart diseases are the most prevalent causes of diseases admitted in hospitals and are the second cause of mortality in Iran. Treatment cost for most of these pre-

Corresponding author: Dr Saeed Bagheri Faradonbeh, bagheri.s@iums.ac.ir

${ }^{1 .}$ Health Management and Economics Research Center, Iran University of Medical Sciences, Tehran, Iran

2. Trauma and Injury Research Center, Iran University of Medical Sciences, Tehran, Iran

3. Department of Cardiology, Hazrat Rasoul Medical and Research Center, Iran University of Medical Sciences, Tehran, Iran

4. Department of Healthcare Management, School of Public Health, Shahid Sadoughi

University of Medical Sciences, Yazd, Iran

5. Firouzabadi Hospital, Iran University of Medical Sciences, Tehran, Iran ventable causes of readmission is remarkably high (1) Ischemic heart disease, a group of heart failure, are categorized into two acute and chronic groups, Ischemic heart

$\uparrow$ What is "already known" in this topic:

PCI strategy was more cost-effective in reducing mortality rate, and in the context of improving QoL, medical therapy strategy was cost-effective.

\section{$\rightarrow$ What this article adds:}

This study may have significant policy and clinical implications for health policymakers, cardiologists, and health managers. 
disease is a condition that results from a decrease in myocardial perfusion. These diseases are often caused by coronary artery occlusion, such as atherosclerosis. Risk factors that develop ischemic heart disease are unhealthy blood cholesterol levels, hypertension, smoking, diabetes mellitus, obesity, lack of physical activity, age and so on. And complications of this disease are Chest pain, left shoulder pain, shortness of breath and weakness $(2,3)$.

Cardiovascular disease is one of the top 10 causes of death in the world, accounting for 25 to 45 percent of the world's mortality as the leading cause of death and the fifth leading cause of disability. 17/3 million people worldwide died in 2008 due to cardiovascular disease, which accounts for about $30 \%$ of all deaths. 80 percent of these deaths occur in poor and middle-income countries. The same death rate is expected to reach 23.6 million by 2030 (4-6). Economic evaluation of cardiovascular interventions plays an important role in decisions about reimbursement, pricing of health care, providing clinical guidance on the use of existing clinical technologies, strategic acquisition of these interventions, providing targeted health care, and generating scientific evidence to make policy decisions and ultimately allocate optimal financial resources to the health of the cardiovascular disease area $(7,8)$.

There are several methods for treating ischemic heart disease, which include: Medical therapy, such as the use ACE inhibitors, $\beta$-blockers, Angiotensin receptor blockers, Mineral corticoid receptor antagonists, Angiotensin receptor neprilysin inhibitor, diuretics, antiplatelet, and lipid regulating drugs. Coronary angioplasty or PCI method that is used to open the closed arteries of the heart. In angioplasty, a small balloon is temporarily inserted into the closed area of the artery to help widen it (9-11).

According to studies, the global cost of cardiovascular diseases in 2010 was estimated at $\$ 863$ billion (average per capita cost of $\$ 125)$, which will grow by $22 \%$ to $\$$ 1044 billion by 2030 and it is predicted that Cardiovascular disease is a major contributor to the disability of active individuals that can severely affect the productivity of active labor force in society and reduce GDP and national income (12).

Percutaneous coronary intervention (PCI) and medical therapy are widely performed as treatment for ischemic heart disease. The cost of this interventions are much so it is essential to consider the economic evaluation of PCI and medical therapy for ischemic heart disease (7). Kodera et al concluded that in ST-elevation myocardial infarction patients, PCI was cost-effective compared to medical therapy (7); and Harindra et al concluded that In patients with stable coronary artery disease, an initial bare-metal stent (BMS) strategy is cost-effective (13). It is known that the costs of medical interventions are rising worldwide, regardless of the development of modern medical technology. Economic evaluations have been emerged as an important tool to allocate scarce resources efficiently and rationally. Methods of economic evaluations are applied to assess health care programs in many arenas containing treatments of ischemic heart disease which impose a high burden on economics worldwide $(14,15)$.
In this study, the costs of interventions under review are identified from the society perspective, including therapeutic and non-therapeutic direct costs and indirect costs. Ouraim was to evaluate the incremental cost-effectiveness rate (ICER) of PCI and medical therapy for patients with ischemic heart disease, using a Markov decision-analytic model.

\section{Methods}

This research has been done in two steps. The first is systematic review and meta-analysis to measure the effectiveness of PCI and medical therapy (mortality rate and quality of life), and the other is a cost-effectiveness analysis. The systematic review stage included observational and interventional studies in English and Persian language from 2000 to 2018 and examined the effectiveness of interventions in patients with ischemic heart disease. Also the quality of all studies is evaluated by the Jadad score (16); meta-analysis was done using RewMan v5 software. We analyzed the results based on the OR. $\mathrm{P}<0.05$ was statistically significant(17). To test heterogeneity, the $\mathrm{I}^{2}$ test was used and because of the high heterogeneity the random effects method is used.

In the second step, a Markov model has been used to model outcomes for patients with ischemic heart disease. The cycle length was 20 years because the average age difference of patients to life expectancy in Iran is 20 years. Outcomes were a reduction in mortality and an increase in quality of life. The analysis was done from the perspective of society. All outcomes were discounted at 3\% per year. Inclusion criteria were patients with ischemic heart disease with ejection fraction less than $40 \%$. Patients were randomly assigned to a treatment group. Ethics Committee of the Iran University of Medical Sciences approved the study.

The two strategies evaluated were medical therapy and PCI. The simplified schematic of the Markov model structure is shown in Figure 1.Clinical experts opinions and reviewing the literature $(13,18,19)$ were used in order to determine the transmission probability. We arbitrarily defined an intervention "cost-effective" if it was less than three times the GDP per capita and "not cost-effective" if it was higher than three times the GDP per capita. The cost-effectiveness threshold was set according to the official dollar rate in Iran in 2018. One-way and two-way sensitivity analyses were performed on all parameters

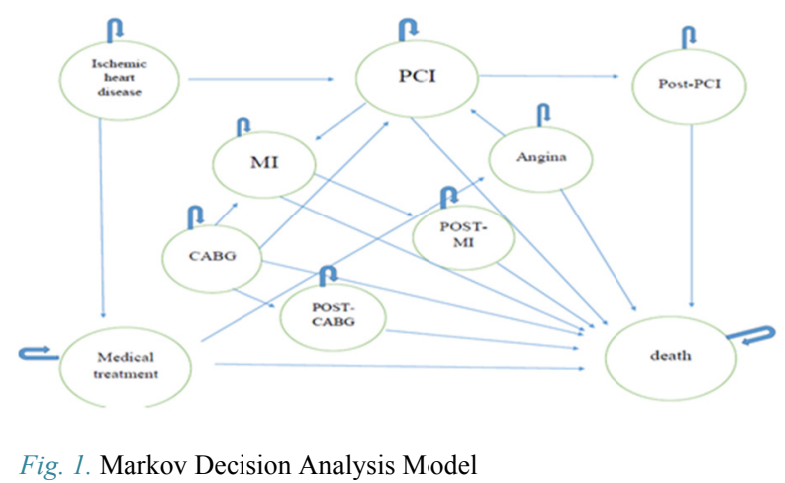


(The costs of two interventions, mortality rate, and quality of life and transition probabilities) including the discount rate from $0 \%$ to $3 \%$.

\section{Results}

In this study, 520 patients with ischemic heart disease were studied that 300 of them were in the PCI group and, 220 of them were in the medical therapy group. Baseline characteristics of the patients are shown in Table 1.

According to Table 1, average age was 53 and 52 years in PCI and MT interventions, respectively. The majority of patients were male $(80 \pm 2)$. On average, $61 \%$ of patients had hypertension, and prevalence of diabetes mellitus in patients was approximately $30 \%, 1 \%$ of patients had dyslipidemia, In both groups, Killip class $\geq 2$ and CCS class $\geq 2$ were estimated at about $25 \%$ and $60 \%$ of patients had Single-vessel disease.

In the systematic and meta-analysis step, study selection procedures are followed according to the PRISMA flow diagram. Finally six full text articles were included in the final analysis (20-25). All the final studies were published between 2000 and 2018. And the random-effects model was selected. In this step experimental and control group were include PCI and MT respectively. The results of first step are summarized in Figure: 2.In the context of this indicator mortality rate in medical group in CI: 0.52-9.68 was 2.23 times the PCI group but this result was not significant in $\mathrm{p}=0.02$.

Also, the results of the study in context to quality of life are shown in Figure 3. The odd ratio of quality of life in medical therapy group in CI: $0.76-1.10$ is 0.91 but this result was not significant in $\mathrm{p}=0.34$.

\section{Cost-effectiveness analysis}

This study was conducted on 520 patients with ischemic heart disease in Iran's referral hospitals in 2018. The costeffectiveness threshold was $\$ 16,482$ and the results of the incremental cost-effectiveness graph were analyzed based on this threshold. The cost-effectiveness plan presented in Figures 4 and 5 and the results of the cost-effectiveness of two interventions in reducing patient mortality and increasing quality of life in these patients are summarized in Table 2.

Table 1. Baseline characteristic

\begin{tabular}{lcc}
\hline Baseline characteristic & PCI & Medical Therapy \\
\hline Average age (years) & 53 & 52 \\
Male (\%) & 80 & 78 \\
Hypertension (\%) & 62 & 60 \\
Diabetes mellitus (\%) & 30 & 28 \\
Dyslipidemia (\%) & 48 & 48 \\
Killip class $^{1} \geq 2(\%)$ & 25 & 26 \\
CCS $^{1}$ class $\geq 2(\%)$ & 24 & 25 \\
Single-vessel disease (\%) & 60 & 60 \\
\hline
\end{tabular}

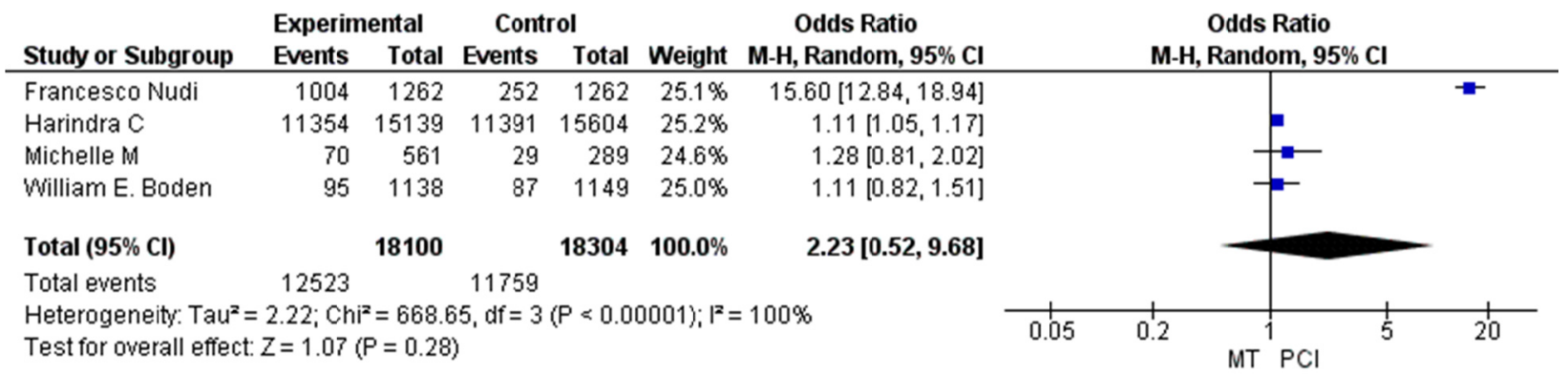

Fig. 2. Forest plot of medical therapy versus percutaneous coronary intervention in mortality rate

\begin{tabular}{|c|c|c|c|c|c|c|c|c|c|}
\hline Study or Subgroup & $\begin{array}{r}\text { MT } \\
\text { Events }\end{array}$ & Total & $\begin{array}{l}\text { PCl } \\
\text { Events }\end{array}$ & Total & Weight & $\begin{array}{c}\text { Odds Ratio } \\
\text { M-H, Fixed, 95\% Cl }\end{array}$ & \multicolumn{3}{|c|}{$\begin{array}{c}\text { Odds Ratio } \\
\text { M-H, Fixed, 95\% Cl }\end{array}$} \\
\hline Michelle M. Graham & 976 & 1302 & 622 & 819 & $81.4 \%$ & $0.95[0.77,1.16]$ & & & \\
\hline Sara Michelly & 139 & 197 & 147 & 194 & $18.6 \%$ & $0.77[0.49,1.20]$ & & & \\
\hline Total $(95 \% \mathrm{Cl})$ & & 1499 & & 1013 & $100.0 \%$ & $0.91[0.76,1.10]$ & & & \\
\hline Total events & 1115 & & 769 & & & & & & \\
\hline $\begin{array}{l}\text { Heterogeneity: } \mathrm{Chi}^{2}= \\
\text { Test for overall effect }\end{array}$ & $\begin{array}{l}.72, d f= \\
z=0.95\end{array}$ & $\begin{array}{l}1(P=0 \\
P=0.3\end{array}$ & $\begin{array}{l}0.40) ; 1^{2}= \\
\text { 4) }\end{array}$ & $0 \%$ & & & 0.5 & $0.7 \mathrm{MT}^{1} \mathrm{PCl}$ & 2 \\
\hline
\end{tabular}

Fig. 3. Forest plot of medical therapy versus percutaneous coronary intervention in quality of life 


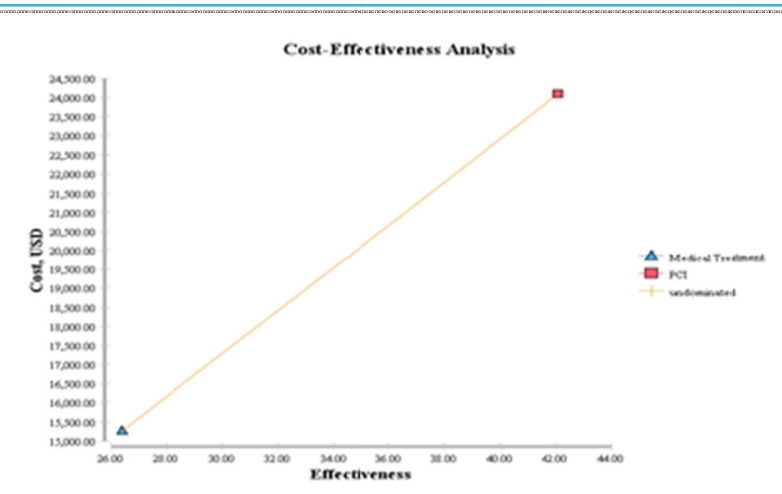

Fig. 4. Cost-Effectiveness plan for reduction in mortality rate

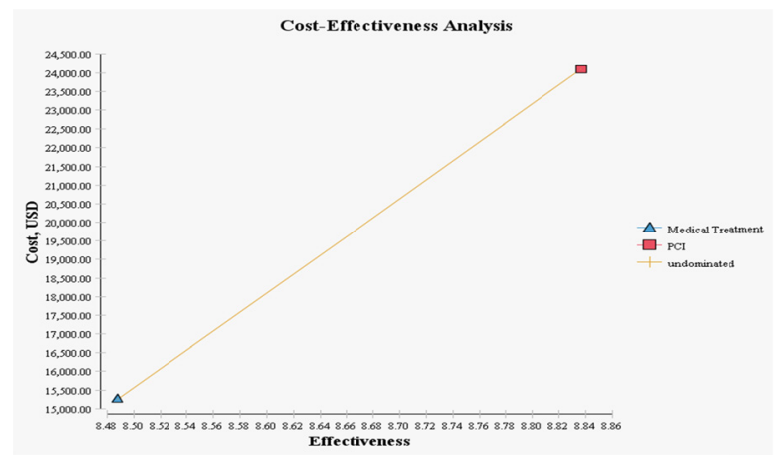

Fig. 5. Cost-Effectiveness plan for Quality of Life

As shown in Table 2, ICER is equal to 562.66 per a unit reduction in mortality rate and Willingness to pay is $\$$ 16,482 , as a result, both interventions are cost-effective but because the average cost-effectiveness of PCI is less than medical therapy, PCI strategy is more cost-effective than medical therapy. Also according to this table, ICER is equal to 25320.11 per a unit of increase in quality of life Therefore; none of the interventions are cost-effective but since the average cost-effectiveness of medical therapy is less than PCI as a result MT strategy is more costeffective than PCI.

\section{Sensitivity analysis}

In this study, first all variables related to the cost and effectiveness of two interventions were selected for sensitivity analysis using the Tornado chart. Then, the variable with the most effect on the study result was selected and, the one-way sensitivity analysis was performed on it. In mortality reduction dimension, the mortality reduction parameter in PCI strategy was selected for analysis and by doing the sensitivity analysis did not change the results of cost-effectiveness. Also in this dimension, the two-way sensitivity analysis did not change the results of cost-

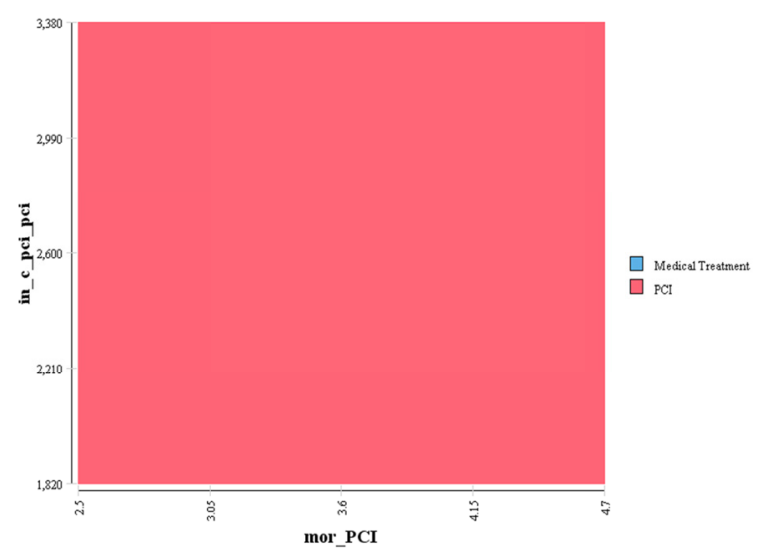

Fig. 6. Two-way sensitivity analysis for reduction in mortality rateindex

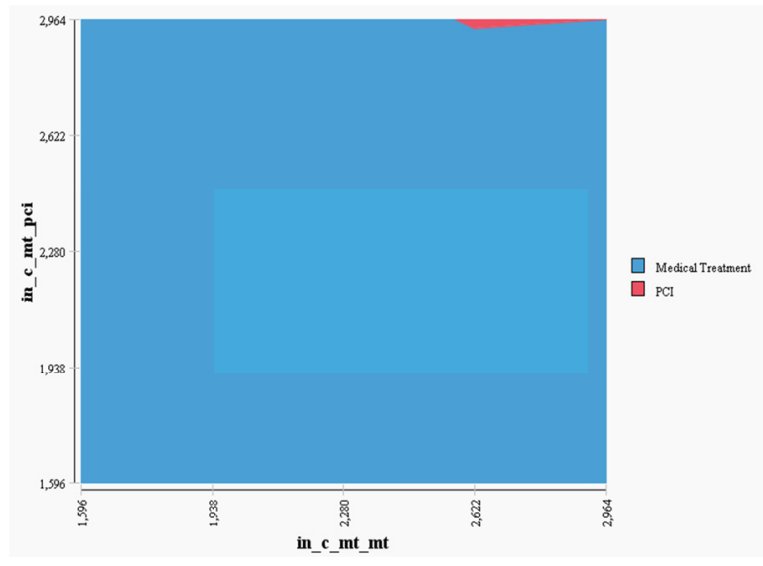

Fig. 7. Two-way sensitivity analysis for Quality of life index

effectiveness (Fig. 6). According to the increasing trend in the quality of life dimension, one-way and two-way sensitivity analysis did not change the results of the costeffectiveness analysis (Fig. 7). Therefore, the model was not sensitive to changing parameters in a specific domain.

\section{Discussion}

In this cost-effectiveness analysis of medical therapy compared to PCI for ischemic heart disease, we constructed a Markov model of treatments for patients in order to compare costs and effectivemess of two interventions based on systematic review and disease costs questionnaire depend on society perspective. We performed a lifetime analysis covering patient life expectancy using a long term period of 20 years. According to the results of present study, PCI strategy is more cost-effective than medical therapy in reduction of mortality rate. Gada et al (26) concluded that PCI is cost-effective in a patient with heart severe symptoms and Quality-of-life criteria, and PCI

Table 2.Cost Effectiveness Analysis

\begin{tabular}{|c|c|c|c|c|c|c|}
\hline Indicator & Intervention & Cost $\$$ & Effectiveness & Average CE & ICER & EV \\
\hline \multirow{2}{*}{ Reduction in mortality } & $\mathrm{MT}$ & 15262.11 & 26.39372 & 578.2476 & 0 & 578.2476 \\
\hline & PCI & 24094.16 & 42.09043 & 572.438 & 562.6691 & 572.438 \\
\hline \multirow{2}{*}{ QoL } & MT & 15262.11 & 8.487849 & 1798.112 & 0 & 1798.112 \\
\hline & PCI & 24094.16 & 8.836665 & 2726.612 & 25320.11 & 2726.612 \\
\hline
\end{tabular}

$\mathrm{MT}=$ medical therapy, $\mathrm{CE}=$ cost effectiveness, $\mathrm{ICER}=$ incremental cost effectiveness ratio, $\mathrm{EV}=$ expected value 
should be used more frequently than medical therapy. Wong et al (27)concluded that PCIis more cost-effective than medical therapy in patients with severe angina. Claude $\mathrm{J}$ et al.(28)concluded that PCI improved clinical effectiveness at only marginally higher costs and was cost-effective; so costs should not be argued against invasive management of elderly patients with chronic angina. Kinlay S (29) concluded that from society's perspective, Percutaneous Transluminal Coronary Angioplasty (PTCA) may be more cost-effective than a medical therapy. So when percutaneous coronary intervention is used, the hospital's financial interests are best retained the use of percutaneous coronary intervention for people with private insurance can be effective. And cost-shifting may have a major impact on the provision of PCI and the costs of providing medical services need to be weighed against the cost of not providing them. The results of all these studies are consistent with the results of the present study.

This study also showed that, considering the increasing quality of life, MT strategy is more cost-effective than the PCI. Griffin et al.(30) in their study on the comparison of the effectiveness of CABG and PCI compared with medical therapy in patients with angina pectoris concluded that medical therapy was a cost-effective strategy in patients under their study. Vieira et al. (31) Concluded that in the long-term economic analysis, medical therapy was more cost-effective than Coronary Artery Bypass Grafting (CABG), and CABG was more cost-effective than PCI for patients with coronary artery disease. The strategy of PCI versus medical therapy was evaluated in the COURAGE trial, which found that PCI reduced angina symptoms and improved the quality of life at 3 years, but did not reduce the rate of death or MI. Besides that, PCI was not costeffective compared with medical therapy (32). Hlatky et al.(33)found that medical therapy in patients with heart failure was more cost-effective compared with PCI Strategy. Ruffo showed that medical therapy dominates PTCA, allowing a greater number of patients to remain free of cardiovascular events at a lower cost (34). The results of all these studies are consistent with the results of the present study

\section{Conclusion}

Cost-effectiveness of PCI in ischemic heart disease must be considered. If PCI does not reduce cardiovascular death or MI when added to medical therapy, it is difficult to justify the cost of PCI for asymptomatic patients. Furthermore, if PCI improves QoL, but does not reduce death, patients and payers need to know how much the cost of a period of freedom from angina is.

The main strength of this economic analysis is that it is based on systematic review and meta-analysis in the field of effectiveness data and that the cost of treating patients was calculated and analyzed from the society perspective and its main limitations were the failure to consider coronary artery bypass surgery intervention and the other limitation is the model-based design. In model-based studies, when model assumptions vary, results vary. So we set reasonable models in the present study based on previous studies and clinical experts opinions.
This cost-effectiveness analysis of medical therapy compared to PCI found that PCI strategy was more costeffective in reducing mortality rate and in the context of improving QoL, medical therapy strategy was costeffective and the high PCI consumables cost highlighted the importance of cost-effective purchasing mechanism. The present study has the following clinical implication: PCI for ischemic heart disease should be performed more often in Iran, based on its cost-effectiveness in the reduction of mortality rate. This study may have significant policy and clinical implications for health policymakers, cardiologists and health managers.

\section{Acknowledgment}

Part of this article was part of a Ph.D thesis in Health Economics at Iran University of Medical Sciences (IUMS), (grant number: IUMS/SHMIS 9321504003). The authors would like to thank Iran University of Medical Sciences for providing financial support for this study.

\section{Conflict of Interests}

The authors declare that they have no competing interests.

\section{References}

1. Farshidi H. Causes of readmission in ischemic heart disease patients. Hormozgan Med J. 2004;8(2):67-71.

2. Homocysteine Studies C. Homocysteine and risk of ischemic heart disease and stroke: a meta-analysis. JAMA. 2002;288(16):2015-22.

3. Rezapour A, Faradonbeh SB, Alipour V, Yusefvand M. Effectiveness of revascularization interventions compared with medical therapy in patients with ischemic cardiomyopathy: A systematic review protocol. Medicine. 2018;97(10)

4. Dababneh E, Goldstein S. Chronic Ischemic Heart Disease, Selection of Treatment Modality. StatPearls [Internet]: StatPearls Publishing; 2018.

5. Investigators WMPP. The World Health Organization MONICA Project (monitoring trends and determinants in cardiovascular disease): a major international collaboration. J Clin Epidemiol. 1988;41(2):10514.

6. Marmot M, Elliott P. Coronary heart disease epidemiology: from aetiology to public health: Oxford Medical Publications; 2005.

7. Satoshi K, Hiroyuki M, Arihiro K. Cost-Effectiveness of Percutaneous Coronary Intervention Compared With Medical Therapy for Ischemic Heart Disease in Japan. Circulation. 2019;83:1498-505.

8. Edlin R, McCabe C, Hulme C, Hall P, Wright J. Cost effectiveness modelling for health technology assessment: Springer; 2015.

9. Faradonbeh SB, Azar FEF, Rezapour A, Hajahmadi M, Hajmiresmaili SJ. Comparing the effectiveness of revascularization interventions with medical therapy in patients with ischemic cardiomyopathy: A systematic review and meta-analysis. Med J Islam Repub Iran. 2018;32:127.

10.Elgendy IY, Mahtta D, Pepine CJ. Medical therapy for heart failure caused by ischemic heart disease. Circ Res. 2019;124(11):1520-35.

11.Greene SJ, Fonarow GC, DeVore AD, Sharma PP, Vaduganathan M, Albert NM, et al. Titration of Medical Therapy for Heart Failure With Reduced Ejection Fraction. J Am Coll Cardiol. 2019;73(19):2365-83.

12.Bloom DE, Cafiero E, Jané-Llopis E, Abrahams-Gessel S, Bloom LR, Fathima S, et al. The Global Economic Burden of Noncommunicable Diseases. Geneva: World Economic Forum; 2012.

13. Wijeysundera HC, Tomlinson G, Ko DT, Dzavik V, Krahn MD. Medical therapy v. PCI in stable coronary artery disease: a costeffectiveness analysis. Med Decis Making. 2013;33(7):891-905.

14.Detsky AS, Naglie IG. A clinician's guide to cost-effectiveness analysis. Ann Intern Med. 1990;113(2):147-54.

15.Eddy DM. Cost-effectiveness analysis: a conversation with my father. JAMA. 1992;267(12):1669-75.

16.Arab-Zozani M, Mahdavi-Mazdeh M, Hasanpoor E, Nejad DG, 
Sokhanvar M, Kakemam E. Safety and Efficacy of Two Different Doses of Everolimus in Kidney Transplantation: a Systematic Review and Meta-Analysis. Iran J Kidney Dis. 2017;11(1):1.

17.Zozani MA, Hosseini SA, Sari AA, Mahdavi-Mazdeh M, Majdzadeh SR, Velayati A. Safety and effectiveness of everolimus compared with sirolimus and tacrolimus in preventing kidney transplantation rejection: a systematic review and meta-analysis. Tehran Univ Med J. 2015;73(5).

18.Scuffham P. Use of fluvastatin following percutaneous coronary intervention. Expert Rev Pharm Out. 2005;5(2):113-23.

19.Scuffham PA, Kósa J. The cost-effectiveness of fluvastatin in Hungary following successful percutaneous coronary intervention. Cardiovasc Drugs Ther. 2006;20(4):309-17.

20.Wijeysundera HC, Bennell MC, Qiu F, Ko DT, Tu JV, Wijeysundera $\mathrm{DN}$, et al. Comparative-effectiveness of revascularization versus routine medical therapy for stable ischemic heart disease: a populationbased study. J Gen Intern Med. 2014;29(7):1031-9.

21.Nudi F, Procaccini E, Versaci F, Giordano A, Pinto A, Neri G, et al. Impact of coronary revascularization on the clinical and scintigraphic outlook of patients with myocardial ischemia. J Cardiovasc Med. 2017;18(6):404-9.

22.Boden WE, O'Rourke RA, Teo KK, Hartigan PM, Maron DJ, Kostuk WJ, et al. Optimal medical therapy with or without PCI for stable coronary disease. N Engl J Med. 2007;356(15):1503-16.

23.Graham MM, Norris CM, Galbraith PD, Knudtson ML, Ghali WA. Quality of life after coronary revascularization in the elderly. European heart journal. 2006;27(14):1690-8.

24.Graham MM, Ghali WA, Faris PD, Galbraith PD, Norris CM, Knudtson ML. Survival after coronary revascularization in the elderly. Circulation. 2002;105(20):2378-84.

25.Brandão SMG, Hueb W, Ju YT, de Lima ACP, Polanczyk CA, Cruz LN, et al. Utility and quality-adjusted life-years in coronary artery disease: Five-year follow-up of the MASS II trial. Medicine. 2017;96(50).

26. Gada H, Whitlow PL, Marwick TH. Establishing the costeffectiveness of percutaneous coronary intervention for chronic total occlusion in stable angina: a decision-analytic model. Heart. 2012;98(24):1790-7.

27.Wong JB, Sonnenberg FA, Salem DN, Pauker SG. Myocardial revascularization for chronic stable angina: analysis of the role of percutaneous transluminal coronary angioplasty based on data available in 1989. Ann Intern Med. 1990;113(11):852-71.

28. Claude J, Schindler C, Kuster GM. Cost-Effectiveness of Invasive Versus Medical Management of Elderly Patients With Chronic Symptomatic Coronary Artery Disease: Findings of the Randomized Trial of Invasive Versus Medical Therapy in Elderly Patients With Chronic Angina (TIME). ACC Curr J Rev. 2005;5(14):28.

29.Kinlay S. Cost-effectiveness of coronary angioplasty versus medical treatment: the impact of cost-shifting. Aust Nz J Med. 1996;26(1):206.

30.Griffin SC, Barber JA, Manca A, Sculpher MJ, Thompson SG, Buxton MJ, et al. Cost effectiveness of clinically appropriate decisions on alternative treatments for angina pectoris: prospective observational study. BMJ. 2007;334(7594):624.

31.Vieira RDO, Hueb W, Hlatky M, Favarato D, Rezende PC, Garzillo $\mathrm{CL}$, et al. Cost-effectiveness analysis for surgical, angioplasty, or medical therapeutics for coronary artery disease: 5-year follow-up of medicine, angioplasty, or surgery study (MASS) II trial. Circulation. 2012;126(11_suppl_1):S145-S50.

32. Weintraub WS, Boden WE, Zhang Z, Kolm P, Zhang Z, Spertus JA, et al. Cost-effectiveness of percutaneous coronary intervention in optimally treated stable coronary patients. Circ Cardiovasc Qual Outcomes. 2008;1(1):12-20.

33.Hlatky MA, Boothroyd DB, Melsop KA, Brooks MM, Mark DB, Pitt $\mathrm{B}$, et al. Medical costs and quality of life 10 to 12 years after randomization to angioplasty or bypass surgery for multivessel coronary artery disease. Circulation. 2004;110(14):1960-6.

34.Ruffo P, Campanella S, Bustacchini S, Mantovani LG. pcv7 economic evaluation of the atorvastatin versus revascularization treatment study (AVERT): anitalian customization. Value Health. 2002;5(6):480. 